\title{
SESSÃO ESPECIAL
}

\section{CÓDIGO DISCIPLINAR Y HISTORIA DE LAS DISCIPLINAS ESCOLARES: ENTREVISTA CON RAIMUNDO CUESTA ${ }^{1}$}

DOI: http://dx.doi.org/10.1590/2236-3459/75183

\begin{abstract}
DISCIPLINARY CODE AND HISTORY OF SCHOOL DISCIPLINES: INTERVIEW WHIT RAIMUNDO CUESTA
\end{abstract}

\author{
Marcelo Pinheiro Cigales \\ Ana Martina Baron Engerroff \\ Universidade Federal de Santa Catarina (Ufsc), Florianópolis/Santa Catarina, Brasil
}

$\cos 8$

\section{Apresentação}

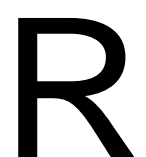

aimundo Cuesta es licenciado en Filosofía y Letras por la Universidad de Salamanca, y doctor en Historia, con premio extraordinario. Ha sido profesor de esa misma disciplina en el IES Fray Luis de León de Salamanca entre 1975 y 2011 (catedrático y jefe de departamento), y profesor colaborador e invitado de la Universidad de Salamanca y de otras universidades españolas y latinoamericanas. Cofundador en 1981 del grupo Cronos en el ICE de la Universidad de Salamanca. Fue director del Centro de Profesores de Salamanca entre 1987 y 1990 . Es miembro fundador de Fedicaria, federación estatal española de grupos de pensamiento crítico en el campo de la educación, que se fundó en 1995. Desde finales de los años noventa su preocupación intelectual más continuada trata de unir la historia de la educación y del curriculum con los

\footnotetext{
${ }_{1}^{1}$ Agradezco a la profesora Gabriela Ossenbach (UNED) que proporcionó el primer contacto con Raimundo Cuesta para la realización de esta entrevista.
} 
fundamentos de una didáctica crítica de las ciencias sociales. A tal fin coordinó, dentro de Fedicaria, un programa de investigación, el Proyecto Nebraska, que se inició con cinco investigaciones sobre la sociogénesis de los códigos disciplinares (de la didáctica como campo, del conocimiento del medio como disciplina, de la psicopedagogía como tecnología de normalización, etc.) y los usos pedagógicos en los modos de educación del mundo contemporáneo. Entre las numerosas publicaciones propias o en colaboración con otros autores, destacan sus libros Sociogénesis de una disciplina escolar: la Historia (Pomares-Corredor, Barcelona, 1997), Clío en las aulas. La enseñanza de la historia en España entre reformas, ilusiones y rutinas (Akal, Madrid, 1998); y Felices y escolarizados. Crítica de la escuela en la era del capitalismo (Octaedro, Barcelona, 2005); Los deberes de la memoria en la educación (Octaedro, Barcelona, 2007); Retazos, memorias y relatos del bachillerato. El Instituto Fray Luis de León de Salamanca 1931-2009 (Salamanca, 2010); "Memoria, historia y educación: genealogía de una singular alianza", en C. Lomas (coord.). Lecciones contra el olvido: educación de la memoria y memoria de la educación (Octaedro, Barcelona, 2011). Una síntesis literaria y autobiográfica de su actividad profesional y vital se puede leer en Las lecciones de Tersites (2017), libro que se ha publicado muy recientemente.

\section{Profesor Raimundo Cuesta, ¿nos podría relatar acerca de su trayectoria profesional, en cuanto profesor e investigador ligado a la historia de las disciplinas escolares?}

Mi formación tuvo lugar en la Universidad de Salamanca y mi vida profesional ha permanecido desde entonces unida a esa ciudad, aunque mi infancia y adolescencia transcurrieron en Santander, una ciudad costera del norte de España en la que nací en 19512. La Universidad de Salamanca, una de las más antiguas de Europa, celebrará el próximo año el ochocientos aniversario de su fundación. En esta localidad pequeña en tamaño pero plagada de monumentos artísticos, y dotada de una numerosa población estudiantil, que supone en torno al veinte por ciento de los 150.000 habitantes de toda la ciudad. Me licencié en Filosofía y Letras con la especialidad de Geografía, Historia y Arte en 1973. Eran los tiempos finales de la dictadura del general Franco y muchos estudiantes nos opusimos a ese ominoso régimen político. Al acabar mis estudios hice una investigación de demografía histórica aplicada la ciudad de Santander en el siglo XVIII.

En 1975, tras ese paréntesis de investigación, tuve la opción de elegir entre una beca de investigación universitaria en el departamento de Historia Moderna de la Universidad de Salamanca o entrar directamente a dar clases de historia en un Instituto de Bachillerato (a alumnos comprendidos entre los catorce y los diecisiete años). Elegí esto último porque me daba mayor independencia económica y personal. No me arrepiento. He sido profesor de Geografía e Historia en el Instituto Fray Luis de Salamanca, fundado a mediados del siglo XIX, entre 1975 y mi jubilación docente en 2011, salvo cuatro años en los que estuve dedicado (tres de ellos) a la formación del profesorado como director del

\footnotetext{
2 Recientemente he publicado un balance de mi vida profesional en Las lecciones de Tersites. Semblanza de una vida y una época (1951-2016). Madrid, Vision Libros, 2017. Hay edición en papel y electrónica, que pueden conseguir en plataformas como Amazon.
} 
Centro de Profesores de Salamanca y uno con licencia por estudios. Por lo demás, en cuanto a mi faceta investigadora, he sido una especie de free lance.

Durante los primeros años de profesión, de 1975 a 1981, mi dedicación fue más militante que investigadora. Dediqué mi tiempo a luchar por una salida democrática de la dictadura franquista durante la llamada Transición, y muy especialmente fui cofundador de la Federación Sindical de las Comisiones Obreras de Enseñanza. Pasada esa fiebre político-sindical, mis intereses giraron hacia la renovación pedagógica. En 1981 fui cofundador del Grupo Cronos con el que obtuvimos numerosas distinciones en España, entre otras el Primer Premio "Francisco Giner de los Ríos" a la Innovación Educativa en 1984. Todo ello coincidió con la reforma educativa llevada a cabo por el socialismo español en los años ochenta. La experiencia dentro del grupo Cronos fue decisiva durante al menos veinte años. Me obligó a ampliar mis horizontes y mi preparación intelectual. Yo que era historiador de formación inicial, ahora frecuenté y estudié otras materias vinculadas a la educación y así fui convirtiéndome en especialista en didáctica de la historia y las ciencias sociales. De esa faceta surgió una amplia gama de materiales didácticos para su empleo en el aula.

Siempre esa actividad estuvo unida al perfeccionamiento permanente del profesorado. Colaboré durante muchos años, con la Universidad de Salamanca, en los diversos programas de formación de docentes. Incluso fui elegido por el profesorado en 1987 director de Centro de Profesores de Salamanca (CEP).

No obstante, las actividades de renovación pedagógica y de formación de profesores no acabaron de satisfacer mis ansias de comprender cómo funcionaba realmente la escuela y el conocimiento que en ella se imparte. Por añadidura, el desencanto con las reformas educativas experimentadas me empujaba también en la misma dirección. Así es cómo se opera el giro hacia una historia de la educación capaz de dotarnos de armas críticas para luchar contra y dentro de las instituciones escolares. En los años noventa me convertí en una rara especie de investigador por libre (sin apoyos económicos ni académicos), jugando al mismo tiempo dentro y fuera de las instituciones universitarias. Esta condición mía extrainstitucional siempre generó sorpresa en mis visitas a universidades latinoameriocanas.

Este largo preámbulo, conviene para entender cómo desde los años noventa, hasta el día de hoy, ocupo la parte más sustancial de mi tiempo a una pesquisa sobre la escuela y las disciplinas escolares. Toda esa investigación acude y se alimenta de múltiples fuentes teóricas y variados campos disciplinares: historia social y nueva sociología del currículum, tradiciones marxistas británicas, estudios culturales, Teoría Crítica de la escuela de Fráncfort, método genealógico Nietzsche-Foucault, sociología de Weber-BourdieuBernstein-Lerena, etc. Siendo la teoría en cualquier investigación, para fraseando a Bourdieu, como "el aire que se respira", mi trabajo de las tres últimas décadas hubiera sido impensable sin ese acompañamiento imprescindible.

2. En su tesis doctoral titulada Sociogénesis de una disciplina escolar: la Historia (1997), usted desarrolla el concepto de código disciplinar para designar un conjunto de ideas, valores, suposiciones y rutinas, que legitiman la función 


\section{educativa atribuida a la Historia. ¿Nos podría hablar más acerca de este concepto? ¿Es posible investigar la historia de otras disciplinas escolares con ese concepto?}

En realidad, así se titula uno de los dos libros (el más célebre) que aparecieron a partir de la tesis (mi tesis y la mayoría de mis obras tienen libre acceso en la página: www.nebraskaria.es)

Ese concepto lo hice público antes de presentar la tesis, en un curso de verano de la Universidad de Santiago de Compostela en 1992. Para mi tal herramienta conceptual fue un hallazgo imprescindible, una llave heurística que me permitió abrir la caja negra de las materias de enseñanza a través del desciframiento del código de la historia escolar. Esa llave me ayudó a desarrollar una perspectiva distinta al ordinario tratamiento de las materias de enseñanza. Ahora bien, no hay conceptos imprescindibles en la investigación social. Los conceptos son invenciones que "pone" el investigador para atrapar una realidad siempre compleja. Eso no quiere decir que valga cualquier conceptualización porque unas enriquecen y otras empobrecen la comprensión de lo que realmente han sido las materias de enseñanza en su larga historia institucional.

Si bien se mira, el concepto de código disciplinar, lo vengo pensando desde hace algún un tiempo, guarda algunos parecidos con el foucaultiano de dispositivo. Comparte con él el hecho de reunir y manifestar una sedimentación histórico-cultural de discursos, artefactos y prácticas. Es, pues, una reunión de discursos y prácticas sociales que interactúan entre sí.

Evidentemente es posible estudiar las otras disciplinas con ese concepto. Así, por cierto, lo hemos hecho dentro del Proyecto Nebraska con el estudio sociogenético del área del conocimiento del medio en la educación primaria, que dio lugar a la tesis de Julio Mateos, cuya síntesis en forma de libro apareció con el título Genealogía de un saber escolar: el código pedagógico del entorno (Barcelona, Octaedro, 2011). Además, tengo noticia que, sobre todo en América Latina, se viene haciendo un amplio uso de mi conceptualización, aunque, como suele ocurrir con los préstamos conceptuales, no siempre se comprenda del todo bien el significado que yo quise dar al sintagma.

Mi descubrimiento y uso del concepto código disciplinar vino a responder a la necesidad de entender de manera diferente la problemática relación entre disciplinas académicas universitarias y disciplinas escolares, es decir, entre el lugar donde se produce un conocimiento (por ejemplo, los historiadores profesionales vinculados a universidades y centros de investigación) y el lugar en el que ese conocimiento sufre una transformación, una alquimia, diría T. S. Popkewitz. Esa alquimia se produce en la enseñanza a través de la práctica profesional docente apoyada en una serie de mediadores (autores de libro de texto, expertos en didáctica, supervisores, programas de estudio, etc.). En la obra de B. Bernstein se explica muy certeramente esa metamorfosis, que está en la base del "misterio" de las materias de enseñanza. Estas son productos culturales originales formados históricamente y no meras copias de un saber académico ya hecho. Recuerdo perfectamente cómo a finales de los ochenta leí mucha historia del pensamiento geográfico siguiendo el rastro de un proyecto dirigido en España por el geógrafo Horacio Capel, que pretendía analizar el devenir de los paradigmas geográficos y juzgar la distancia entre la geografía investigada y la enseñada. Pero, como demuestra la historia de la enseñanza de la historia, las disciplinas escolares no son espejos de paradigmas científicos (aunque, en 
parte, también pueden serlo). Por tanto, el estudio del código disciplinar nos autoriza a estudiar formulaciones discursivas y textos visibles, pero también aquellas prácticas institucionales que permanecen ocultas y que, sin embargo, son estratégicas a la hora de comprender la lógica evolutiva de las materias de enseñanza.

El concepto de código disciplinar, en tanto que tradición sociocultural de larga duración, me ayudó a entender las materias escolares como productos originales, no naturales, como realidades históricas resultantes de un complejo haz de poderes (el Estado, los grupos profesionales, los intereses políticos de cada momento, el marcado, etc.), que, al final, generan la recontextualización en el marco de la institución escolar del conocimiento socialmente disponible. Al respecto, mis deudas con Bernstein, Goodson, Chervel, Lundgren y otros son abundantes. Como complemento y marco, te remito a un artículo de Antonio Viñao, cuya primera versión data de 2006 y la que cito es una reactualización más reciente escrita para la revista Pro-Posições.

\section{3. ¿Cómo ve usted el papel de los manuales escolares en la constitución del código disciplinar?}

Los manuales escolares son la punta del iceberg de las disciplinas escolares. Yo los tildé de "textos visibles" (junto a los programas escolares) del código disciplinar. En varios países existen excelentes programas de recopilación y estudio de los libros de texto. En España está el proyecto Manes. Yo mismo utilicé ciento de manuales sobre la enseñanza de la historia para componer mi tesis. Incluso me convertí en coleccionista de los textos de historia que configuraron el origen de la historia como materia de enseñanza. A menudo cuento cómo un libro de Historia de España, escrito en los años treinta del siglo XVIII por un jesuita francés preceptor de los hijos del rey español Felipe V, llegó a convertirse, tras la consiguiente reducción (Sumario de la historia de España en verso), en un molde de la historia escolar del siglo XIX.

La investigación convencional sobre estos interesantes artefactos culturales tiende a ver en ellos la plasmación, más o menos retardada, de la evolución de las ciencias de referencias y también de cosmovisiones ideológicas. Pero esta es una mirada muy limitada de la cuestión. El libro de texto no es solo una criatura de una ciencia referente o de un mundo ideológico circundante, es, a efectos de la enseñanza, una entidad cosificada si no se pone en contacto con el contexto institucional de su uso en las clases. Por señalar dos factores, las tradiciones profesionales o el carácter examinador (evaluable y medible) del saber escolar trasmutan y dan sentido al empleo del texto. Todo texto escolar necesita, pues, una hermenéutica del contexto en el que se desenvuelve. Eso requiere estudiar las disciplinas escolares valiéndonos también de otras fuentes complementarias como pueden ser exámenes escolares, reglamentos, testimonios de los protagonistas, observaciones directas o indirectas de lo que ocurre en las aulas, etc. Es decir, los manuales son textos inertes que requieren iluminarlos poniéndolos en contacto con el contexto real de la práctica de la enseñanza. Los profesores y las profesoras, sus prácticas de enseñanza, son los textos vivos.

Todo esto, claro, si entrar en lo que Apple llamó la "economía política" del libro de texto, o sea, sin considerar la dimensión mercantil que poseen los materiales de enseñanza, cada vez más en nuestro tiempo. En parte, eso explica su larga e inextinguible vida. Desde 
luego, también los libros de texto ejercen un efecto espejo mediante el que se manifiestan las ideologías y los estereotipos sociales dominantes en cada época.

4. En el libro Clío en las aulas (1998), usted propone una "Didáctica Crítica de la Historia". ¿En qué consiste esta didáctica crítica? ¿Ella pasa necesariamente por la integración de las disciplinas, o sea, por una "Didáctica Crítica de las Ciencias Sociales?"

En ese libro, en efecto, esbozo lo que podría ser una didáctica crítica de las ciencias sociales, que luego he ido desarrollando más amplia y matizadamente en nuestra revista Con-Ciencia Social. Esta publicación pertenece a Fedicaria, una plataforma española de pensamiento crítico, asociación que cofundé en 1995. En 1999, en esa revista (que es de libre acceso en www.fedicaria.org) publiqué un artículo que puede decirse "fundacional" de un proyecto colectivo de pensar críticamente la enseñanza de la historia y de las ciencias sociales. Allí expuse los postulados de una didáctica (problematizar el presente, pensar históricamente, educar el deseo, aprender dialogando e impugnar los códigos socioprofesionales), siempre atenta a los problemas sociales más que a la supuesta lógica disciplinar de los programas escolares al uso. Resumiéndolo, lo que luego llamé de diversas maneras (una historia del presente, una historia genealógica o una historia con memoria), apunta hacia a un conocimiento histórico de los problemas que nos afectan. Obsérvese que tal planteamiento se hacía a raíz precisamente de mis aportaciones a la historia de las disciplinas escolares. Esa investigación y mi profesión siempre fueron de la mano. Durante los ocho años anteriores a mi jubilación, entre 2003 y 2011, llevé a cabo en mi centro el llamado programa de Los deberes de la memoria en la educación (también es el título de un libro mío), esto es, una concreción de lo que yo entendía por uso público de la historia dentro de las instituciones escolares.

\section{En qué momento surgió la idea del proyecto Nebraska, ¿podría hacernos un relato de este proyecto y sus líneas de investigación?}

El proyecto Nebraska nace en 2002 dentro de Fedicaria y se mantiene vivo hasta 2012 (su historia detallada y la mayoría de sus publicaciones de sus miembros son de libre acceso en www.nebraskaria.es). Los profesores de Fedicaria más afines a mis investigaciones sobre el código disciplinar y los modos de educación, convenimos en profundizar en ellos mediante investigaciones particulares que eran intercambiadas en reuniones periódicas. Esos afanes se plasmaron, entre otros muchos resultados, en dos brillantes tesis doctorales. El disparador o banco de pruebas de todo ese proyecto fue, desde 2002 la discusión, según lo iba elaborando, de mi libro Felices y escolarizados. Crítica de la escuela en la era del capitalismo (Barcelona; Octaedro, 2005). Las tesis de Juan Mainer, presentada en la Universidad de Zaragoza en 2007 (véase una versión amplia en La forja de un campo profesional. Pedagogía y didáctica de las ciencias sociales en España. Madrid, CSIC, 2009) y la de Julio Mateos leída en 2008 en la Universidad Salamanca (véase una versión muy sucinta en Genealogía de un saber escolar: del código pedagógico del entorno. Barcelona, Octaedro, 2011), profundizaron y desarrollaron nuevos horizontes en una perspectiva teórica compartida en Nebraska. 
Lo que más quiero destacar es que proyecto Nebraska, nacido dentro de Fedicaria, no solo era un plan de investigación contrahegemónica en lo ideológico, sino también en lo institucional. El extraño nombre de "Nebraska" proviene de la cafetería de Madrid en la que, tomando unas cervezas, fundamos este proyecto Juan Mainer, Julio Mateos y yo. Lo que no quita que estos trabajos acabaran teniendo un formato universitario o académico. Igual que con Fedicaria demostramos que es posible pensar contracorriente en plataformas autónomas y libres toda servidumbre económica o de otro estilo.

Sería prolijo explicar con detalle las líneas precisas de cada una de las indagaciones. Estas como los universos conceptuales y metodológicos fueron una profundización en mi primitiva tesis de 1997 y su concreción puede estudiarse con detalle en la citada página de www.nebraskaria.es

6. Pensando en la lógica de los campos sociales, de los que nos habla Pierre Bourdieu, y las disciplinas escolares, ¿es posible decir que en el campo educacional las disciplinas escolares generan unos conocimientos propios acerca del mundo social, que comparados con sus respectivos campos científicos, se parecen poco a ellos?

Creo que ya he expuesto, en respuestas anteriores, el carácter original e idiosincrásico de las disciplinas escolares. Ahora bien, me interesa decir que los conceptos fundamentales del sociólogo Pierre Bourdieu, los de "campo" y "habitus", empapan mi obra y la del proyecto Nebraska, aunque siempre con una enorme libertad interpretativa y sin guardar ortodoxias de ninguna clase. Para orientar al lector o lectora de esta entrevista, quiero recomendar una obra reciente en la que se manejan los conceptos-clave del proyecto Nebraska, a saber, código disciplinar, modos de educación, campo profesional y habitus. Se trata del trabajo que escribí con Juan Mainer en la revista Historia y Memoria de la Educación (n. 1, 2014, p. 351-393) titulado Guardianes de la tradición y esclavos de la rutina: historia del campo profesional de los catedráticos de Instituto.

7. En un texto de Viñao (2000, p. 461) (VIÑAO FRAGO, Antonio. La catalogación de los manuales escolares y la historia de las disciplinas a través de sus denominaciones. In: TIANA FERRER, Alejandro (Coord.). EI libro escolar, reflejo de intenciones políticas e influencias pedagógicas. Madrid: Universidad Nacional de Educación a Distancia, 2000. p. 451-469), en el que propone catalogar los textos escolares haciendo uso de las denominaciones históricas de las disciplinas escolares, hemos visto que la Sociología surge como disciplina escolar en la enseñanza secundaria en España junto a la disciplina de Filosofía. Viñao recoge dos denominaciones que incluyen a la Sociología y que se dieron al inicio del siglo XIX: Sociología y ciencias éticas (1894) y Ética y Sociología (1900). En su opinión, ¿por qué la Sociología no se convirtió en disciplina escolar en la enseñanza secundaria en España?

Bueno, la madeja de los cambios continuos de planes de estudio en la historia del bachillerato español es célebre. Apareció alguna vez, en efecto, el nombre "sociología" 
vinculada a la ética, pero en realidad ese título es poco significativo y más bien la materia matriz siempre fue la Filosofía, que ha sido una de las asignaturas "fuertes", es decir, parte del tronco curricular más duradero en la enseñanza secundaria. En realidad, la Geografía y la Historia en la escuela estuvieron siempre en el centro de la dimensión social del currículo. Hasta finales de siglo, en 1899, no se crea en España una cátedra de Sociología en la Facultad de Filosofía y Letras destinada a los cursos de doctorado, que ocupó Manuel Sales Ferré, un eminente krausista de la tradición liberal progresista. En España hasta 1972 no se establece la licenciatura de Sociología como una realidad autónoma dentro de la Facultad de Ciencias Políticas y Sociología. De modo y manera, que la sociología como tal, es un ser extraño en la historia de la educación española. Recuerdo, no obstante, que estudié en el "preuniversitario", durante el curso 1966-1967, algo como doctrina social de la Iglesia católica, cuya dogmática se revestía con algunos oropeles científicos.

Pienso que la consideración muy cerrada y tradicionalmente disciplinar de la Geografía y la Historia no dejó demasiados huecos para otras ciencias sociales. Solo tras la Ley General de Educación de 1970 se crea un área de Ciencias Sociales y sobre todo tras la Ley General de Ordenación del Sistema Educativo de 1990 se debatió extensamente si los programas debían ser de Historia-Geografía o de Ciencias Sociales. Al final, más allá de los rótulos los profesores de sociales en la educación secundaria provienen de las facultades de Historia. En la primaria es algo diferente, pero en lo fundamental lo social reproduce en parte el viejo código disciplinar de la historia.

Las tradiciones disciplinares, a pesar de una creciente homogeneización de los lenguajes y los contenidos curriculares, no son similares en todos los países. Por ejemplo, en Estado Unidos se concedió más importancia a los estudios sociales. También en algunos países de América Latina. No obstante, en los tiempos de la "modernización conservadora" de Margaret Thatcher en Gran Bretaña o de EEUU en los años ochenta se pretendió regresar a las asignaturas de "siempre", entre otras la historia. En España, sobre todo en la educación secundaria, el profesorado tiene casi como patrimonio y seña de identidad su formación en las facultades de Historia. En mi país la Sociología nace y permanece encerrada dentro de la Facultad de Ciencias Políticas (creada en 1944 por el régimen de Franco para dotarse de elites directivas). Su comparecencia en estudios no universitarios es más bien marginal. La razón de que esto haya sido así se debe a que el curriculum no deja de ser un campo de fuerzas confrontadas en el seno del cual las comunidades científicas pugnan por abrirse paso y legitimarse mediante su aterrizaje en las aulas. En España, como en Francia, los historiadores han ganado siempre la partida. En todo caso, lo que se imparte actualmente en las aulas es una mezcla de tecnicismo pedagógico e historia tradicional. Pervive una porción nada despreciable del código disciplinar, que en el siglo XIX se inventaba al tiempo que se levantaba el edificio del Estado liberal-nacional y de su correspondiente sistema educativo.

Estimo que, a la hora de estudiar las disciplinas escolares, es aconsejable ver qué es lo que se esconde bajo el nombre de las materias de enseñanza que figura en los programas escolares. Hay que ver lo que ocurre debajo de la punta del iceberg. La mejor ciencia social es siempre la que no se limita a describir las apariencias. Hay que buscar aquello que no se ve a primera vista y por ello es preciso radiografiar el código disciplinar de las asignaturas escolares. En fin, en la historia de la educación española la Sociología ha sido una no-disciplina escolar. 
Finalmente, me gustaría añadir algunas consideraciones complementarias a propósito de la deriva del campo de estudio de las investigaciones sobre la historia de las disciplinas escolares en España. Aunque mis trabajos más recientes ya no han versado sobre las disciplinas escolares, mi legado en este tema ha sido más citado que empleado en los círculos académicos hispanos. Me parece que en América Latina he tenido una mayor influencia real. Incluso en los últimos años se aprecia en mi país una suerte de revisionismo que pone el acento en la dimensión supuestamente sociologista, ideológica y poco atenta a la libertad del profesorado de mi propuesta sociocrítica. Esta revisión se hace en nombre de la libertad el profesorado y de otros supuestos de la acción racional y libre de los individuos frente a las instituciones sociales. El regreso hacia posiciones antisociológicas se presenta como algo creativo cuando no es más que una excrecencia ideológica de un neoliberalismo rampante en los círculos académicos, invadidos por un capitalismo académico y un individualismo metodológico de notables proclividades anglosajonas. En 2018 tengo pensado colaborar con M. A. Pereyra, T. Popkewitz, J. Romero y otros destacados especialistas en un monográfico sobre los supuestos teóricos que han informado, desde un punto de vista crítico, la historia del curriculum. Sin duda, será un momento para recapitular y seguir mirando hacia adelante.

\section{8. ¿Qué le parece el cultivo de la historia de la educación en Brasil? ¿Qué relación mantiene usted con los estudiosos de ese país?}

El campo de la historia de la educación brasileño guarda más de un paralelismo con el devenir del mismo en España y en los países latinoamericanos. A pesar de que ni he estado nunca en ese inmenso y espléndido país ni me considero un experto en sus pesquisas educativas, lo cierto es que las comparaciones son evidentes. La Sociedad Brasileña de Historia de la Educación (SBHE) nace en 1995 (la SEDHE, la sociedad española, doce años antes) en el contexto de un proceso que se inicia en la mayoría de los países latinoamericanos en los años noventa. Por ese tiempo surge también la Revista Brasileira História da Educação (RBHE). Hoy la sociedad brasileña se encuentra integrada en la ISCHE (International Standing Conference for the History of Education) y constituye una "potencia" nacional por su número de afiliados y pos sus actividades. Revistas como la que publica esta entrevista, Revista História da Educação / History of Education Journal, acreditan el fortalecimiento y el vigor de un campo de estudio relativamente joven.

Mi relación con el mundo académico brasileño ha sido muy escasa. Entre 2000 y 2009 impartí en la Universidad de Salamanca docencia sobre Sociogénesis del conocimiento escolar dentro del programa de doctorado Perspectiva histórica, comparada y política de la educación. En ese curso predominaron los alumnos procedentes de América Latina, dentro de los destacaron, por sus inquietudes y formación, algunos profesores y estudiantes brasileños, con los que mantuve una relación muy amigable y fructífera, que en algunos casos se prolongó posteriormente. Ignoro si esa relación tendió un puente para el conocimiento de mi obra en Brasil.

Hace unos años hice una prospección sobre la difusión de mi concepto de código disciplinar y comprobé que en Colombia, Brasil y Argentina su extensión era apreciable. En el caso de Brasil, además de algunos alumnos míos del doctorado, juzgo, a modo de hipótesis por confirmar, que pudo tener alguna importancia un artículo de uno de los más 
brillantes historiadores españoles de la educación. En efecto, Antonio Viñao Frago publicó en 2008 en la RBHE (Revista Brasileira da Educação) un texto sobre las disciplinas escolares y los diversos enfoques, en el que mi obra aparecía muy subrayada y muy bien valorada.

Desde luego, mi conocimiento de la producción brasileña acerca de historia de las disciplinas escolares no es ni mucho menos completa, pero sí me parece atisbar que las fuentes teóricas con las que se trabaja me resultan muy próximas. Además observo que se recurre a historiadores franceses de primera importancia, como André Chervel o Dominique Julia, rompiendo con el absurdo hábito de utilizar y citar solo aquello que se escribe en inglés. En mi caso, en Brasil, la lengua no ha sido una barrera. No obstante, me gustaría señalar que la recepción de mi obra en América Latina (he sido invitado a universidades y centros de investigación de Colombia, México, Chile y Argentina) no se circunscribe a la historia de la educación, sino también a la didáctica de la Historia. También esto ocurre en Brasil; valga como ejemplo el quehacer, en la última década, de María Auxiliadora Moreira dos Santos a propósito de la historia de la enseñanza de la historia en Brasil o Ana Claudia Urban acerca del estudio de los manuales escolares de historia. En ambos casos se maneja mi concepto de código disciplinar. Por cierto, en los próximos meses seré miembro de un tribunal de doctorado en la Universidad de Alcalá de Henares (Madrid) que juzga la tesis de una profesora brasileña, Luciana Oliveira Correia, con la que me entrevisté en el curso de su indagación sobre la plasmación de la historia en los manuales escolares. En todo caso mi visión del campo académico brasileño es muy parcial e "interesada". Quizás la de alguien que está al mismo tiempo fuera y dentro del mundo universitario de la historia de la educación.

\section{Breve selección bibliográfica de Raimundo Cuesta}

CUESTA, Raimundo. Clío en las aulas: la enseñanza de la Historia en España entre reformas, ilusiones y rutinas. Madrid: Ediciones Akal, 1998.

Felices y escolarizados. Crítica de la escuela en la era del capitalismo. Barcelona: Octaedro, 2005.

Genealogía y cambio conceptual. Educación, historia y memoria. Archivos Analíticos de Políticas Educativas, v. 22, n. 23, p. 1-25, 2014.

. La venganza de la memoria y las paradojas de la historia. Salamanca: Lulu.com, 2015.

Las lecciones de Tersites, Semblanza de una vida y una época (1951-2016). Madrid: Visión Libros, 2017.

Sociogénesis de una disciplina escolar: la Historia. Barcelona: Pomares Corredor, 1997.

CUESTA, Raimundo; MAINER, Juan. Guardianes de la tradición y esclavos de la rutina: historia del campo profesional de los catedráticos de instituto. Historia y Memoria de la Educación, n. 1, p. 351-393, 2014.

CUESTA, Raimundo; MAINER, Juan; MATEOS, Julio (Coords.). Reformas y modos de educación en España: entre la tradición liberal y la tecnocracia. Monográfico de la Revista Andorra, n. 11, p. 18-94, 2011. 
Transiciones, cambios y periodizaciones en la historia de la educación.

Fedicaria/Ceince. Salamanca: Lulu.com, 2009.

MARCELO PINHEIRO CIGALES é Licenciado em Ciências Sociais e Mestre em Educação pela Universidade Federal de Pelotas. Durante a graduação realizou intercâmbio junto à Faculdade de Economia da Universidade de Coimbra. Atualmente, é doutorando em Sociologia Política pela Universidade Federal de Santa Catarina, com período de pesquisa junto ao Centro de Investigación Manuales Escolares (Manes) da Universidad Nacional de Educación a Distancia (Uned-Madrid), financiado pela Capes/PDSE (n. 88881.134645/201601).

Endereço: Rua Cecília dos Santos, 147, casa 04 - 88040450 - Florianópolis/SC - Brasil.

E-mail: marcelo.cigales@gmail.com

ANA MARTINA BARON ENGERROFF é Mestra em Sociologia Política pela Universidade Federal de Santa Catarina, onde atualmente é doutoranda na mesma área, com bolsa Capes.

Endereço: Rua José Victor da Rosa, 722, ap. 1404B - 88117-405 - São José/SC - Brasil.

E-mail: anambaron@hotmail.com

Recebido em 23 de julho de 2017.

Aceito em 21 de agosto de 2017. 